\title{
Path Planning and Vehicle Scheduling Optimization for Logistic Distribution of Hazardous Materials in Full Container Load
}

\author{
Huo Chai, ${ }^{1,2}$ Ruichun He, ${ }^{2}$ Changxi Ma, ${ }^{2}$ Cunjie Dai, ${ }^{1,3}$ and Kun Zhou ${ }^{1}$ \\ ${ }^{1}$ Mechatronics Technology and Research Institute, Lanzhou Jiaotong University, Lanzhou 730070, China \\ ${ }^{2}$ School of Traffic and Transportation, Lanzhou Jiaotong University, Lanzhou 730070, China \\ ${ }^{3}$ Engineering Technology Center for Information of Logistics \& Transport Equipment, Lanzhou 730070, China \\ Correspondence should be addressed to Ruichun He; tranman@163.com
}

Received 4 July 2017; Revised 30 September 2017; Accepted 26 October 2017; Published 16 November 2017

Academic Editor: Luisa Di Paola

Copyright (C) 2017 Huo Chai et al. This is an open access article distributed under the Creative Commons Attribution License, which permits unrestricted use, distribution, and reproduction in any medium, provided the original work is properly cited.

\begin{abstract}
Mathematical models for path planning and vehicle scheduling for logistic distribution of hazardous materials in full container load (FCL) are established, with their problem-solving methods proposed. First, a two-stage multiobjective optimization algorithm is designed for path planning. In the first stage, pulse algorithm is used to obtain the Pareto paths from the distribution center to each destination. In the second stage, a multiobjective optimization method based on Nondominated Sorting Genetic Algorithm II (NSGA-II) is designed to obtain candidate transport paths. Second, with analysis on the operating process of vehicles with hazardous materials in FCL, the vehicle scheduling problem is converted to Vehicle Routing Problem with Time Windows (VRPTW). A problem-solving method based on estimation of distribution is adopted. A transport timetable for all vehicles based on their transport paths is calculated, with participation of the decision-makers. A visual vehicle scheduling plan is presented for the decision-makers. Last, two examples are used to test the method proposed in this study: distribution of hazardous materials in a small-scale test network and distribution of oil products for sixteen gas stations in the main districts of Lanzhou city. In both examples, our method is used to obtain the path selection and vehicle scheduling plan, proving that validity of our method is verified.
\end{abstract}

\section{Introduction}

Similar to that of ordinary goods, logistic distribution of hazardous materials can be classified as full container load (FCL) $[1,2]$ and less than container load (LCL) [3]. If the transport volume required by a destination is no less than the capacity of one transportation vehicle, there will be no less than one vehicle involved in a logistic job. To efficiently finish the job, operation of vehicles in FCL is necessary. Distribution of goods in LCL can be attributed to the commonly seen Vehicle Routing Problem (VRP). To plan for the logistic distribution of ordinary goods in FCL, the generalized shortest paths between the distribution center and multiple destinations can be adopted. However, optimization of distribution of hazardous materials involves at least two objectives: transportation cost and risk. In such scenario, multiple nondominated paths between the distribution center and the destinations exist. As a result, a vehicle transport path has to be chosen according to risk preferences in a specific situation.

The problem of path planning and vehicle scheduling for logistic distribution of hazardous materials in LCL is fundamentally the multiobjective VRP [4]. Zografos and Androutsopoulos established a Vehicle Routing Problem with Time Windows (VRPTW) model for distribution of hazardous materials and designed an insertion-based biobjective heuristic algorithm. On such basis, they introduced a model for the issue of emergency site selection and designed a Lagrangian relaxation heuristic algorithm [5]. Their contributions constituted a full set of decision support system for transport path planning of hazardous materials and coordinated emergency response arrangement after accidents. In a case study of natural gas transportation in Thailand, Pradhananga et al. proposed a genetic algorithm, converting the HAZMAT 
Vehicle Routing Problem with Time Windows (HVRPTW) with multiple objectives, namely, transportation cost and risk, into a single objective problem through linear weighting [6]. Later, they proposed a multiobjective ant colony algorithm targeting at solving this problem, the validity of which was verified in a case of distribution of liquefied petroleum gas in Osaka, Japan [7].

Existing studies on path planning and vehicle scheduling for logistic distribution of hazardous materials in FCL focus on transportation of hazardous materials between an origin and a destination (OD), while those between a distribution center and multiple destinations are rarely seen. In this study, the problem-solving of logistic distribution of hazardous materials in FCL is divided into two stages: path planning and vehicle scheduling. First, a multiobjective optimization model targeting at transportation cost and risk is established for path planning. However, if existing evolutionary multiobjective optimization algorithms are adopted, all the nodes along the transportation network have to be encoded. By doing so, the extended length of code in the case of largescale transportation networks will result in large volumes of calculation, which leads to difficulty in finding the optimal solution under limited number of iterations. To avoid such situation, we design the two-stage method mentioned. In the first stage, a pulse algorithm is used to obtain the Pareto-optimal paths from the distribution center to each destination, so that only the Pareto-optimal paths obtained are allowed as candidate solutions subsequently. In the second stage, the Pareto-optimal paths obtained in the first stage are encoded to calculate the final results using algorithms based on Nondominated Sorting Genetic Algorithm II (NSGA-II). By segmenting the problem into subproblems in two stages, the volume of calculation is significantly reduced, which leads to an increased efficiency in calculation and correspondingly raises probability of obtaining the optimal solution. Then, based on the path plan selected by decision-makers, each distribution job of a destination is treated as a node in VRP, with time window of the node being the time window of the destination. By doing so, vehicle scheduling problem is converted to a single objective VRPTW. An algorithm based on Univariate Marginal Distribution Algorithm (UMDA) is designed to solve the VRPTW, to obtain a transport schedule for all vehicles.

\section{Mathematical Model}

To solve the distribution problem of vehicles with hazardous materials in FCL, optimizations are carried out in both path planning and vehicle scheduling stages, with a purpose of minimizing transportation cost and total risk of all transportation jobs. To simplify the risk evaluation process of transportation of hazardous materials, two assumptions are proposed on the premise of not violating practical conditions.

(1) Risks produced for vehicles with the same type in the same road section are equivalent. The risks are relevant only to conditions of a specific road section, without considering other factors such as whether loaded volume of a vehicle reaches its capacity and vehicle conditions.
(2) The risk value of a vehicle transporting with no loads is zero. That is, when a vehicle returns to the distribution center after unloading hazardous materials in a destination site, it chooses a path with the minimum transportation cost.

In a transport network $G=(N, E), N$ represents a set of $n$ nodes and $E$ represents a set of road sections between these nodes. The potential risk for a vehicle of a certain type transporting hazardous materials in a road section $(i, j)$ is $r_{i j}$. The transportation cost induced can be classified into two cases: cost of $c_{i j}^{1}$ for transport with FCL when the travel time is $\Delta t_{i j}^{1}$ and cost of $c_{i j}^{2}$ for transport with no load when the travel time is $\Delta t_{i j}^{2}$. Assume there are $m$ destinations $\left(D=\left\{d_{1}\right.\right.$, $\left.\left.d_{2}, \ldots, d_{m}\right\}, D \subset N\right)$ to distribute hazardous materials to them. Each destination requires a transport volume of $g_{1}, g_{2}, \ldots, g_{m}$, with a time window of $\left[E T_{d}, L T_{d}\right]$ for destination $v$. There are multiple paths $P_{o d}$ from the distribution center $o$ to the destination $d$. All vehicles start from the distribution center $o$ and return to the distribution center after finishing its job. The capacity of each vehicle is $q$. The average loading time and unloading time for a vehicle are $\Delta t^{1}$ and $\Delta t^{2}$, respectively. Variable $\delta_{i j}^{o d}=1$ indicates $(i, j) \in E$; namely, the road section is in the path from the distribution center $o$ to the destination $d$; otherwise, $\delta_{i j}^{o d}=0$. Subsequently, we analyze the distribution time, number of vehicles, as well as the total traveling distance of vehicles, and the total risk.

2.1. Number of Vehicles. Distribution of vehicles with FCL, namely, $g_{d} \geq q$, is mainly concerned in this section. In this condition, a vehicle can either finish the whole job or commit a portion of a job. The number of vehicles required by a destination is $a_{d} . a_{d}$ can be determined in the following rules:

(1) When $g_{d} / q$ is an integer, $a_{d}=g_{d} / q$.

(2) When $g_{d} / q$ is not an integer, $a_{d}=\left\lceil g_{d} / q\right\rceil$.

The total number of vehicles required to finish distribution jobs of all destinations is $\sum_{d} a_{d}$. The number of vehicles required on the condition of given required transport volume of a destination is constant. Although the total number of vehicles is constant, the same vehicle is allowed to participate in distribution jobs of multiple destinations when the condition of time window is satisfied. Issues concerning the number of vehicles dispatched and schedules for vehicles leaving/arriving will be discussed in the section of vehicle scheduling optimization.

2.2. Total Cost and Risk of Vehicle Travel. To finish the distribution job of destination $d, a_{d}$ vehicles have to be involved. The transportation cost of all vehicles from the distribution center to the destinations is the sum of transportation cost of all road sections traveled, namely,

$$
c_{o d}^{1}=a_{d} \sum_{(i, j)} c_{i j}^{1} \delta_{i j}^{o d} .
$$

Based on assumption (2), the returning path of a vehicle with no load from the destination to the distribution center is the 
path with minimum cost, represented as $c_{o d}^{2}$, with its value calculated using the shortest path algorithm. According to the analysis above, the transportation cost involved in the distribution job for the destination $d$ is $c_{o d}^{1}+c_{o d}^{2}$.

Similarly, the total risk involved in the distribution job of destination $d$ is

$$
r_{o d}=a_{d} \sum_{(i, j)} r_{i j} \delta_{i j}^{o d}
$$

2.3. Vehicle Path Optimization. The following mathematical model (P1) of vehicle path optimization can be established:

$$
\begin{gathered}
\text { P1: } \min \quad f_{1}=\sum_{d \in N} a_{d}\left(\sum_{(i, j) \in E} c_{i j}^{1} \delta_{i j}^{o d}+c_{o d}^{2}\right) \\
\min \quad f_{2}=\sum_{d \in N} a_{d} \sum_{(i, j) \in E} r_{i j} \delta_{i j}^{o d} \\
\text { s.t. } \quad \sum_{j} \delta_{i j}^{o d}-\sum_{j} \delta_{j i}^{o d}= \begin{cases}1 & i=o \\
-1 & i=d \\
0 & \text { otherwise }\end{cases} \\
\forall j \in N, o \in N, d \in N \\
\delta_{i j}^{o d}=\{0,1\} \quad \forall o, d \in N,(i, j) \in E .
\end{gathered}
$$

The objective function (3) is a functional expression of transportation cost, and the objective function (4) is a functional expression of transportation risk. Constraint (5) ensures the formation of a complete transportation path from the distribution center to destinations.

2.4. Distribution Time. The travel time of a vehicle from the distribution center to destination $d$ and its corresponding returning time are, respectively,

$$
\begin{aligned}
& \Delta t_{o d}^{1}=\sum_{(i, j)} \Delta t_{i j}^{1}, \\
& \Delta t_{o d}^{2}=\sum_{(i, j)} \Delta t_{i j}^{2} .
\end{aligned}
$$

The time used by a vehicle for a round trip from the distribution center to the destination $d$ and, after it finishes distribution, back to the distribution center is in total

$$
\Delta t_{o d}=\Delta t^{1}+\Delta t_{o d}^{1}+\Delta t^{2}+\Delta t_{o d}^{2}
$$

Assuming the leaving time of a vehicle from the distribution center is $t_{o d}^{0}$, the time moment when the vehicle arrives at the distribution center from destination $d$ should be $t_{o d}^{0}+\Delta t_{o d}$. Since the required time window for destination $d$ is $\left[E T_{d}, L T_{d}\right]$, the time moment when the vehicle arrives at the destination $d$ should satisfy the following condition:

$$
E T_{d} \leq t_{o d}^{0}+\Delta t^{1}+\Delta t_{o d}^{1} \leq L T_{d}
$$

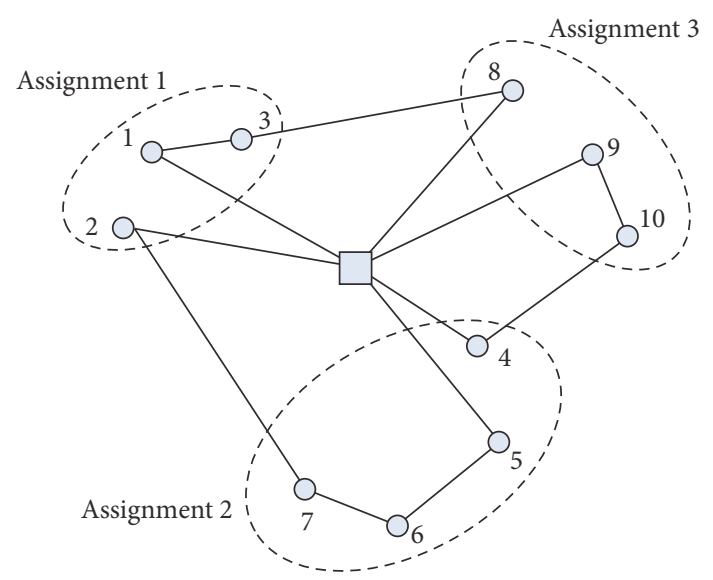

FIgURE 1: A sketch diagram of vehicle scheduling assignments converted to VRPTW.

2.5. Vehicle Scheduling. By solving model $\mathrm{P} 1$, the vehicle travel paths from the distribution center to various destinations and back to the distribution center are obtained. Decision-makers are allowed to choose favorable travel paths depending on the specific risk preferences, though how to determine a vehicle dispatching plan is still an issue to be addressed. With limited vehicles available, the number of vehicles for dispatching is an optimization objective to be considered.

For convenience, each distribution job for a destination is divided into several assignments; namely, a distribution for a vehicle is considered as an assignment. Any one assignment should be finished within the time range from the earliest starting time to the latest ending time. Each assignment should be undertaken by only one vehicle. Each vehicle undertakes a new assignment only after it finishes an assignment on hand. Each assignment can be viewed as a node in a two-dimensional plane. All the destinations can be arranged according to the serial numbers of nodes. In this way, each assignment in a distribution job can be thus numbered. For example, the assignments for the distribution job to destination $d_{1}$ can be represented by $J_{d_{1}}=\left\{1,2, \ldots, a_{d_{1}}\right\}$; assignments for $d_{2}$ can be represented by $J_{d_{2}}=\left\{a_{d_{1}}+1, a_{d_{1}}+\right.$ $\left.2, \ldots, a_{d_{1}}+a_{d_{2}}\right\}$; assignments for $d_{3}$ can be represented by $J_{d_{3}}=\left\{a_{d_{1}}+a_{d_{2}}+1, a_{d_{1}}+a_{d_{2}}+2, \ldots, a_{d_{1}}+a_{d_{2}}+a_{d_{3}}\right\}$. $J=J_{d_{1}} \cup J_{d_{2}} \cup \cdots \cup J_{d_{m}}$ represents the set of all assignments. The vehicle scheduling problem can be converted to VRP with time windows (Figure 1), with the objective function indicating a balance between minimum number of vehicles and average minimum loads carried by each vehicle. The time window and service time for assignment $i\left(i \in J_{d}\right)$ are $\left[E T_{d}-\right.$ $\left.\left(\Delta t^{1}+\Delta t_{0 d}^{1}\right), L T_{d}+\left(\Delta t^{2}+\Delta t_{0 d}^{2}\right)\right]$ and $t_{0 d}$, respectively. Since a node carries only information of assignments, transport volume is not contained and can be regarded as zero, and no restriction is posed on the capacity of vehicles.

$x_{i j v}\left(x_{i j v} \in[0,1]\right)$ is used to indicate whether a vehicle $v$ continues with assignment $j$ after it finishes assignment $i$. If yes, $x_{i j v}=1$, else $x_{i j v}=0 . y_{i v}\left(y_{i v} \in[0,1]\right)$ is used to 


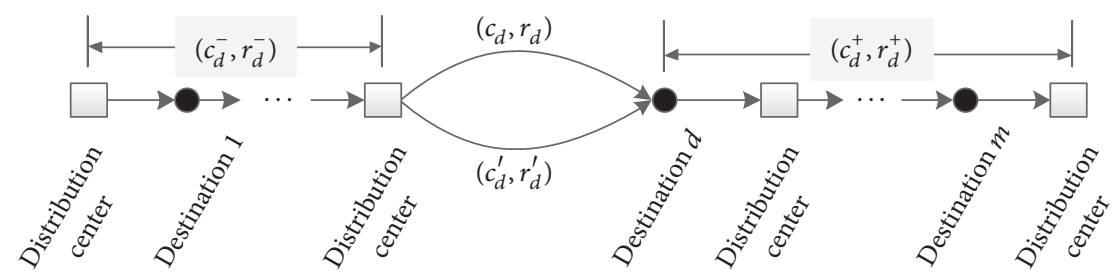

FIGURE 2: Total cost and total risk of path formed by connecting the distribution center with each destination.

indicate whether assignment $i$ is implemented by vehicle $v$. If yes, $y_{i v}=1$, else $y_{i v}=0$. The vehicle scheduling problem can be described by the following model (P2):

$$
\begin{array}{ll}
\text { P2: min } & f \\
& =M \sum_{i \in J} \sum_{v \in V} x_{0 i v}+\sum_{v \in V}\left(\sum_{i \in J} \sum_{j \in J} \Delta t_{i j} x_{i j v}-\mu\right)^{2} \\
\text { s.t. } & \sum_{v \in V} y_{i v}=1 \quad \forall i \in J, \\
& \sum_{i \in J} x_{i j v}=y_{j v} \quad \forall j \in J, \quad v \in V, \\
& \sum_{j \in J} x_{i j v}=y_{i v} \quad \forall i \in J, \quad v \in V, \\
& \sum_{i \in J} x_{i j k}-\sum_{j \in J} x_{i j k}=0 \quad \forall v \in V, \\
& T_{j v}=T_{i v}+\Delta t_{0 d} x_{i j v} \\
\quad \forall d \in N, i \in J_{d}, \quad j \in J, v \in V, \\
\\
& E T_{d}-\left(\Delta t^{1}+\Delta t_{0 d}^{1}\right) \leq T_{j v} \\
& \leq L T_{d}+\left(\Delta t^{2}+\Delta t_{0 d}^{2}\right) \\
& \quad \forall d \in N, j \in J_{d}, v \in V, \\
& x_{i j v} \in[0,1] \quad \forall i, j \in J, v \in V, \\
& y_{i v} \in[0,1] \quad \forall i \in J, \quad v \in V .
\end{array}
$$

The objective function (11) represents the minimum number of vehicles and difference of their travel time, where $\mu=\sum_{v \in V} \sum_{i \in J} \sum_{j \in J} \Delta t_{i j} x_{i j v} / \sum_{i \in J} \sum_{v \in V} x_{0 i v}$. The first part of this function represents the minimum number of vehicles; $M$ is a sufficiently large integer, to ensure the priority level of the objective of vehicle number. The second part represents minimized standard deviation of travel time of all vehicles under the same number of vehicles, to ensure minimum difference in transport jobs for each vehicle. Constraints (12)-(15) guarantee that each vehicle can carry out only one assignment at a time. That is, assignments can be carried out by a vehicle consecutively, but not in parallel. In addition, an assignment can be carried out by only one vehicle. Constraint (16) represents the time moment when node $j$ is reached. Constraint (17) ensures satisfaction of time window restriction at the moment when node $j$ is reached.

\section{Problem-Solving Method}

The optimization objective of model P1 is to obtain the Pareto-optimal solution sets of total cost and total risk for distribution jobs from the distribution center to all destinations. This process can be viewed as a calculation of transportation cost and risk involved in the path formed by connecting the distribution center to each destination (see Figure 2). $\left(c_{d}^{-}, r_{d}^{-}\right)$ represents the cost and risk of the path before reaching destination $d$ from the distribution center; $\left(c_{d}^{+}, r_{d}^{+}\right)$represents the cost and risk of the path from the destination $d$ back to the distribution center. Assume $\left(c_{d}^{-}+c_{d}+c_{d}^{+}, r_{d}^{-}+r_{d}+r_{d}^{+}\right)$is a solution among the Pareto solution set. If $\left(c_{d}, r_{d}\right)<\left(c_{d}^{\prime}, r_{d}^{\prime}\right)(<$ indicating domination), then $\left(c_{d}^{-}+c_{d}^{\prime}+c_{d}^{+}, r_{d}^{-}+r_{d}^{\prime}+r_{d}^{+}\right)$will not emerge in the Pareto solution set. This conclusion can be simply proved by contradiction. If $\left(c_{d}^{-}+c_{d}^{\prime}+c_{d}^{+}, r_{d}^{-}+r_{d}^{\prime}+r_{d}^{+}\right)$ is one solution among the Pareto solution set, then either $c_{d}^{-}+c_{d}^{\prime}+c_{d}^{+} \leq c_{d}^{-}+c_{d}+c_{d}^{+}$or $r_{d}^{-}+r_{d}^{\prime}+r_{d}^{+} \leq r_{d}^{-}+r_{d}+r_{d}^{+}$ has to hold. Namely, either $c_{d}^{\prime} \leq c_{d}$ or $\leq r_{d}$ should be correct, which contradicts $\left(c_{d}, r_{d}\right) \prec\left(c_{d}^{\prime}, r_{d}^{\prime}\right)$.

As can be seen, in order to obtain the Pareto solution set of model P1, a Pareto-optimal solution is calculated for the path from the distribution center to each destination, so that other paths are excluded out. A two-stage method is adopted.

The problem of finding the path from the distribution center to each destination is essentially a biobjective shortest path problem (BSP). Generally, there are two types of solutions to it: dynamic programming (DP) $[8,9]$ and ranking [10]. Pulse algorithm [11] is a precise algorithm to solve BSP. Although an idea of recursively traversing all paths in a network is adopted, it is still efficient by using a four-step pruning mechanism to exclude dominated paths effectively. Other than the advantage of high efficiency, this algorithm has a virtue of finding a full set of Pareto-optimal solution, which is hardly achievable in heuristic or evolutionary algorithms. The key procedure to pulse algorithm is a newly added node, if satisfying one of the following four conditions, incoming partial path will be pruned, which means the incoming partial path containing this node will be eliminated.

(1) It contains cycles.

(2) It exceeds either one or both upper bounds [11] defined by the nadir point before reaching the end node. 


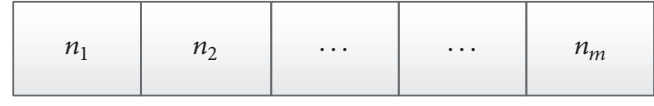

FIgURE 3: Encoding format.

(3) It is dominated by any solution which is in current efficient set before reaching the end node.

(4) Each node that is passed by a feasible path is marked with a label storing two objective values of the path. If the incoming partial path is dominated by either objective value stored in the label, then this path is dropped, or else the objective value of this path will be added in the label of the node.

In the second stage, the Pareto paths obtained in the first stage are encoded to calculate the final results using multiobjective optimization based on NSGA-II [12]. Since no paths other than the Pareto paths are involved in the second stage, the length of code is significantly shrunk when using multiobjective optimization. Therefore, the chance of obtaining the optimal solution increases prominently under the same size of population and number of iterations.

The following introduces encoding method, fitness function, and population regeneration strategy for NSGA-II.

(1) Individual Coding. Natural number is adopted for individual coding, the length of coding is $m$, and Figure 3 shows the format of the encoding.

Where $n_{i} \in\left[1, \max \left(\left|P_{o d_{i}}\right|\right)\right], P_{o d_{i}}$ is Pareto-optimal path set from the distribution center 0 to the destination nodes $d_{i}$. If the $i$ th value of the code is $n_{i}$, then it means $n_{i} \bmod \left|P_{o d_{i}}\right|$ th path (obtained by the first stage) from the distribution center $o$ to the destination node $d_{i}$ is adopted for transportation. For example, the numbers of Pareto-optimal paths from the distribution center 0 to the destination nodes $d_{1} \sim d_{4}$ are 2, 3, 5 , and 4 , respectively. Then a code of $[1,4,3,5]$ means the 1 st (1 $\bmod 2), 1 s t(4 \bmod 3), 3 r d(3 \bmod 5)$, and $1 s t(5 \bmod 4)$ of the Pareto paths from o to $d_{1} \sim d_{4}$ for the individual are adopted for transportation.

(2) Fitness Function. Assuming indi represents an individual, [c_value, $r_{-}$value $]=f($ ind $i)$ is used to represent fitness of the individual ind $i$, where $c_{-}$value and $r_{\text {_vvalue }}$ are transportation cost and risk, respectively, based on the selected path plan of individual indi.

(3) Population Updating Strategy. A population adds in new individuals by crossover, for which integers are used. First, two individuals are randomly selected in the population, with two positions, pos 1 and pos 2 (pos $1<\operatorname{pos} 2$ ), randomly generated. These two individuals, pos 1 and pos 2 , are crossed over to generate two new individuals.

Mutation is also operated with integers to obtain new individuals. An individual is selected randomly, while two positions, pos 1 and pos 2 (pos $1<$ pos 2 ), are randomly generated. Positions of the selected individual, pos 1 and $p o s 2$,
TABLE 1: Lengths of each road section and risks of vehicles traveling in each road section.

\begin{tabular}{lcc}
\hline Road section & Length/km & Risk \\
\hline$(0,1)$ & 36.56 & 0.0456 \\
$(0,3)$ & 9.85 & 0.0214 \\
$(0,4)$ & 30.29 & 0.0154 \\
$(0,5)$ & 15.82 & 0.0286 \\
$(0,6)$ & 9.26 & 0.0059 \\
$(1,2)$ & 45.16 & 0.0217 \\
$(1,3)$ & 30.36 & 0.0020 \\
$(1,4)$ & 45.47 & 0.0083 \\
$(2,3)$ & 24.26 & 0.0232 \\
$(2,5)$ & 27.95 & 0.0225 \\
$(2,7)$ & 39.18 & 0.0252 \\
$(4,6)$ & 27.16 & 0.0186 \\
$(4,8)$ & 48.89 & 0.0068 \\
$(5,7)$ & 21.26 & 0.0546 \\
$(5,8)$ & 36.35 & 0.0032 \\
$(6,8)$ & 24.15 & 0.0457 \\
$(7,8)$ & 42.65 & 0.0022 \\
\hline
\end{tabular}

are subtracted by $\max \left(\left|P_{o d}^{(n)}\right|\right)$ separately, with absolute values taken, to generate a new individual.

Model P2 is targeted at the objective of balance between number of vehicles and transport job intensity undertaken by each vehicle, which is essentially VRP. Except that the transport distance and number of vehicles in the objective function should be replaced by number of vehicles and fairness of transport assignments, other steps can be solved by referring to the problem-solving method for VRPTW. In this study, the problem-solving method based on UMDA for VRPHTW given by literature [13] based on estimation of distribution is adopted.

The problem-solving process for path planning and vehicle scheduling of hazardous materials transportation is shown in Figure 4.

\section{Numerical Example}

4.1. A Small-Scale Network. Figure 5 shows a test transportation network, where node 0 represents the distribution center, and nodes 1, 2, 4, 7, and 8 represent destinations. The distance and risk of each road section in the network are shown in Table 1 . The required transport volume and time window of each destination are shown in Table 2. The capacity of a vehicle is $13.5 \mathrm{~m}^{3}$. The average loading time, unloading time, and the average travel speed of a vehicle are $0.75 \mathrm{~h}, 0.75 \mathrm{~h}$, and $45 \mathrm{~km} / \mathrm{h}$, respectively. The cost for transport in FCL is $50 \$ / \mathrm{km}$; the cost for transport with no load is $10 \$ / \mathrm{km}$. A vehicle transport plan aims to minimize the transportation cost and risk.

Table 2 shows the required transport volumes of all destinations as well as the accepted unloading time windows, which is hard, indicating a waiting action if a vehicle arrives earlier than the earliest starting time specified. 
TABLE 2: Required transport volume and time window of a destination.

\begin{tabular}{lccccc}
\hline Destination & 1 & 2 & 4 & 7 & 8 \\
\hline Required volume $/ \mathrm{m}^{3}$ & 35 & 18 & 29.5 & 45 & 60 \\
Time window & {$[8: 00,16: 00]$} & {$[12: 00,20: 00]$} & {$[8: 00,12: 30]$} & {$[8: 00,18: 00]$} & {$[8: 00,20: 00]$} \\
\hline
\end{tabular}

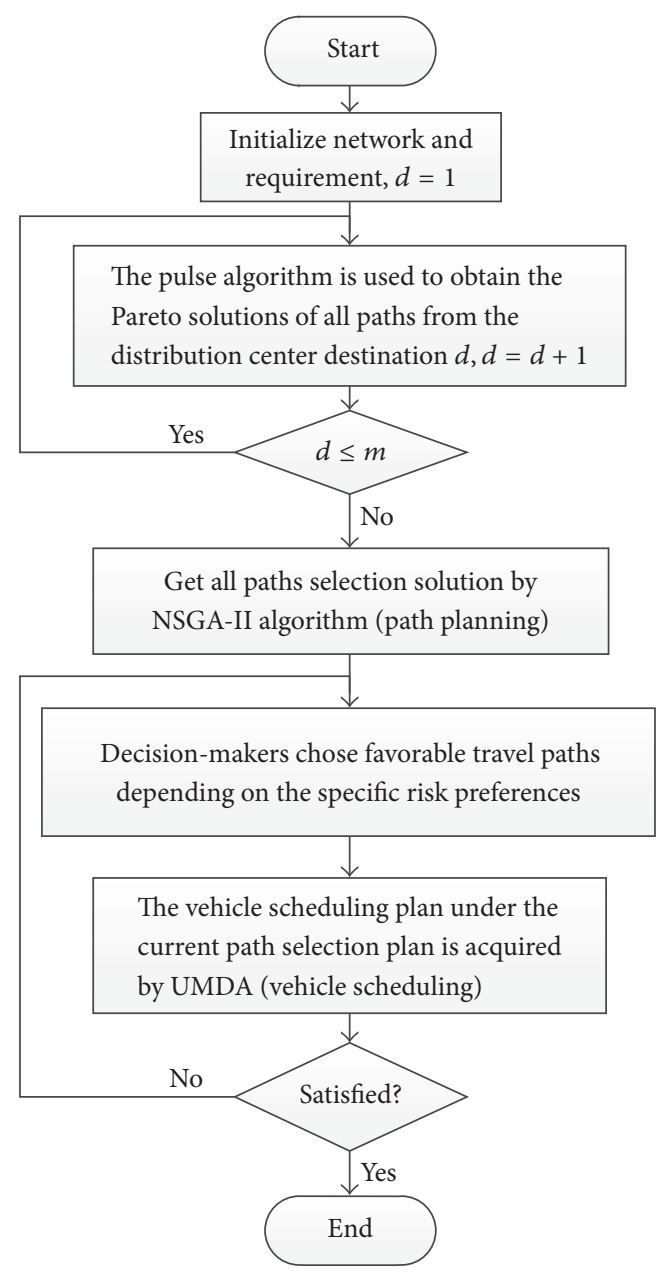

FIGURE 4: Flow chart for solving problem of path planning and vehicle scheduling of hazardous materials transportation.

After the first stage, Pareto shortest path (departing path) and shortest distance paths (returning path) between the distribution center to each destination can be obtained (Table 3).

When the Pareto paths set is obtained, the Pareto solution set of total cost and risk for transportation between the distribution center and all destinations can be obtained. Table 4 shows the path selection plans after decoding of Pareto solutions.

When a certain transport plan is selected, model P2 is used to solve the results for the path adopted. In this way, a vehicle scheduling plan under the current path selection plan is acquired. For example, when path plan 1 is selected in Table 4, the vehicle scheduling plan of Table 5 can be obtained, and when path plan 14 is selected, the vehicle
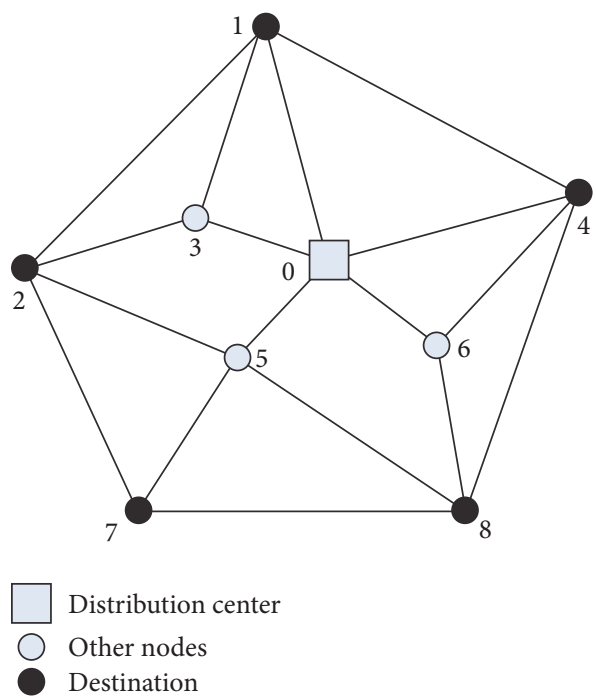

FIGURE 5: A test network for transportation of hazardous materials.

scheduling plan of Table 6 can be obtained. The column of node represents the distribution node arrived at by a vehicle. The timetable shows the time moments of a vehicle in a round trip for loading start (LS), departure from the distribution center (DD), unloading start at the destination (US), returning after unloading finishes (RU), and arriving back at the distribution center (AC).

4.2. Comparison with General NSGA-II. In addition, a general NSGA-II algorithm (GNA) is designed for the path planning in order to illustrate the effectiveness of the twostage algorithm (TSA) proposed. Different from the twostage algorithm, in the general NSGA-II algorithm, the priority based encoding [14] method is adopted, and the encoding length is $|N| * m$. However, in the second stage of the two-stage algorithm, the encoding length is only $m$. For the example in Section 4.1, the parameters of two algorithms are set as follows: the size of population is 100, and the iteration number is 50 generations. In Figures $6(\mathrm{a})-6(\mathrm{~d})$ is the Pareto-optimal front obtained by iterative 5, 10, 20, and 50 generations, respectively. By comparison, it can be found that the final solution can be obtained by using the TSA when the iteration reaches the 10th generation (14 plans, see Table 4) while the GNA still fails to obtain the optimal solution until the fiftieth iteration. This is just a network of 9 nodes and this gap will become even bigger for large-scale networks. There is no direct relationship between the encoding length and the network size in the two-stage algorithm, so, for large-scale networks, the algorithm is still valid. 
TABLE 3: Departing path and returning path between the distribution center and each destination.

\begin{tabular}{|c|c|c|c|c|c|}
\hline Destination & Departing path & Length/km & Risk & Returning path & Length $/ \mathrm{km}$ \\
\hline \multirow{2}{*}{1} & $0 \rightarrow 1$ & 36.56 & 0.0456 & \multirow{2}{*}{$1 \rightarrow 0$} & \multirow{2}{*}{36.56} \\
\hline & $0 \rightarrow 3 \rightarrow 1$ & 40.21 & 0.0234 & & \\
\hline 2 & $0 \rightarrow 3 \rightarrow 2$ & 34.11 & 0.0446 & $2 \rightarrow 3 \rightarrow 0$ & 34.11 \\
\hline 4 & $0 \rightarrow 4$ & 30.29 & 0.0154 & $4 \rightarrow 0$ & 30.29 \\
\hline \multirow{5}{*}{7} & $0 \rightarrow 5 \rightarrow 7$ & 37.08 & 0.0832 & \multirow{5}{*}{$7 \rightarrow 5 \rightarrow 0$} & \multirow{5}{*}{37.08} \\
\hline & $0 \rightarrow 3 \rightarrow 2 \rightarrow 7$ & 73.29 & 0.0698 & & \\
\hline & $0 \rightarrow 6 \rightarrow 8 \rightarrow 7$ & 76.06 & 0.0538 & & \\
\hline & $0 \rightarrow 5 \rightarrow 8 \rightarrow 7$ & 94.82 & 0.0340 & & \\
\hline & $0 \rightarrow 4 \rightarrow 8 \rightarrow 7$ & 121.83 & 0.0244 & & \\
\hline \multirow{3}{*}{8} & $0 \rightarrow 6 \rightarrow 8$ & 33.41 & 0.0516 & \multirow{3}{*}{$8 \rightarrow 6 \rightarrow 0$} & \multirow{3}{*}{33.41} \\
\hline & $0 \rightarrow 5 \rightarrow 8$ & 52.17 & 0.0318 & & \\
\hline & $0 \rightarrow 4 \rightarrow 8$ & 79.18 & 0.0222 & & \\
\hline
\end{tabular}

TABLE 4: Path selection plans.

\begin{tabular}{|c|c|c|c|}
\hline Solution & Path & Total cost $/ \$$ & Total risk \\
\hline 1 & $\begin{aligned} 0 & \rightarrow \mathbf{1} \rightarrow 0 \\
0 \rightarrow 3 & \rightarrow \mathbf{2} \rightarrow 3 \rightarrow 0 \\
0 & \rightarrow \mathbf{4} \rightarrow 0 \\
0 \rightarrow 5 & \rightarrow 7 \rightarrow 5 \rightarrow 0 \\
0 \rightarrow 6 & \rightarrow \mathbf{8} \rightarrow 6 \rightarrow 0\end{aligned}$ & $2,161.32$ & 0.8630 \\
\hline 2 & $\begin{aligned} 0 \rightarrow 3 & \rightarrow \mathbf{1} \rightarrow 0 \\
0 \rightarrow 3 \rightarrow \mathbf{2} & \rightarrow 3 \rightarrow 0 \\
& 0 \rightarrow \mathbf{4} \rightarrow 0 \\
0 \rightarrow 5 & \rightarrow 7 \rightarrow 5 \rightarrow 0 \\
0 \rightarrow 6 & \rightarrow \mathbf{8} \rightarrow 6 \rightarrow 0\end{aligned}$ & $2,185.41$ & 0.7964 \\
\hline 3 & $\begin{aligned} 0 & \rightarrow \mathbf{1} \rightarrow 0 \\
0 \rightarrow 3 & \rightarrow \mathbf{2} \rightarrow 3 \rightarrow 0 \\
0 & \rightarrow \mathbf{4} \rightarrow 0 \\
0 \rightarrow 5 & \rightarrow \mathbf{7} \rightarrow 5 \rightarrow 0 \\
0 \rightarrow 5 & \rightarrow \mathbf{8} \rightarrow 6 \rightarrow 0\end{aligned}$ & $2,367.68$ & 0.7640 \\
\hline 4 & $\begin{aligned} 0 \rightarrow 3 & \rightarrow \mathbf{1} \rightarrow 0 \\
0 \rightarrow 3 \rightarrow \mathbf{2} & \rightarrow 3 \rightarrow 0 \\
0 \rightarrow \mathbf{4} & \rightarrow 0 \\
0 \rightarrow 5 & \rightarrow 7 \rightarrow 5 \rightarrow 0 \\
0 \rightarrow 5 & \rightarrow \mathbf{8} \rightarrow 6 \rightarrow 0\end{aligned}$ & $2,391.77$ & 0.6974 \\
\hline 5 & $\begin{array}{c}0 \rightarrow 3 \rightarrow \mathbf{1} \rightarrow 0 \\
0 \rightarrow 3 \rightarrow \mathbf{2} \rightarrow 3 \rightarrow 0 \\
0 \rightarrow \mathbf{4} \rightarrow 0 \\
0 \rightarrow 5 \rightarrow 8 \rightarrow 7 \rightarrow 5 \rightarrow 0 \\
0 \rightarrow 6 \rightarrow \mathbf{8} \rightarrow 6 \rightarrow 0\end{array}$ & $2,528.43$ & 0.6788 \\
\hline 6 & $\begin{array}{c}0 \rightarrow \mathbf{1} \rightarrow 0 \\
0 \rightarrow 3 \rightarrow \mathbf{2} \rightarrow 3 \rightarrow 0 \\
0 \rightarrow \mathbf{4} \rightarrow 0 \\
0 \rightarrow 5 \rightarrow 8 \rightarrow 7 \rightarrow 5 \rightarrow 0 \\
0 \rightarrow 6 \rightarrow \mathbf{8} \rightarrow 6 \rightarrow 0\end{array}$ & $2,669.43$ & 0.6662 \\
\hline 7 & $\begin{array}{c}0 \rightarrow 3 \rightarrow \mathbf{1} \rightarrow 0 \\
0 \rightarrow 3 \rightarrow \mathbf{2} \rightarrow 3 \rightarrow 0 \\
0 \rightarrow \mathbf{4} \rightarrow 0 \\
0 \rightarrow 5 \rightarrow 7 \rightarrow 5 \rightarrow 0 \\
0 \rightarrow 4 \rightarrow \mathbf{8} \rightarrow 6 \rightarrow 0\end{array}$ & $2,688.88$ & 0.6494 \\
\hline 8 & $\begin{array}{c}0 \rightarrow 3 \rightarrow \mathbf{1} \rightarrow 0 \\
0 \rightarrow 3 \rightarrow \mathbf{2} \rightarrow 3 \rightarrow 0 \\
0 \rightarrow \mathbf{4} \rightarrow 0 \\
0 \rightarrow 5 \rightarrow 8 \rightarrow 7 \rightarrow 5 \rightarrow 0 \\
0 \rightarrow 6 \rightarrow \mathbf{8} \rightarrow 6 \rightarrow 0\end{array}$ & $2,693.52$ & 0.5996 \\
\hline
\end{tabular}


TABle 4: Continued.

\begin{tabular}{|c|c|c|c|}
\hline Solution & Path & Total cost $/ \$$ & Total risk \\
\hline 9 & $\begin{array}{c}0 \rightarrow 3 \rightarrow \mathbf{1} \rightarrow 0 \\
0 \rightarrow 3 \rightarrow \mathbf{2} \rightarrow 3 \rightarrow 0 \\
0 \rightarrow \mathbf{4} \rightarrow 0 \\
0 \rightarrow 6 \rightarrow 8 \rightarrow 7 \rightarrow 5 \rightarrow 0 \\
0 \rightarrow 5 \rightarrow \mathbf{8} \rightarrow 6 \rightarrow 0\end{array}$ & $2,734.79$ & 0.5798 \\
\hline 10 & $\begin{array}{c}0 \rightarrow \mathbf{1} \rightarrow 0 \\
0 \rightarrow 3 \rightarrow \mathbf{2} \rightarrow 3 \rightarrow 0 \\
0 \rightarrow \mathbf{4} \rightarrow 0 \\
0 \rightarrow 5 \rightarrow 8 \rightarrow 7 \rightarrow 5 \rightarrow 0 \\
0 \rightarrow 5 \rightarrow \mathbf{8} \rightarrow 6 \rightarrow 0\end{array}$ & $2,875.79$ & 0.5672 \\
\hline 11 & $\begin{array}{c}0 \rightarrow 3 \rightarrow \mathbf{1} \rightarrow 0 \\
0 \rightarrow 3 \rightarrow \mathbf{2} \rightarrow 3 \rightarrow 0 \\
0 \rightarrow \mathbf{4} \rightarrow 0 \\
0 \rightarrow 5 \rightarrow 8 \rightarrow \mathbf{7} \rightarrow 5 \rightarrow 0 \\
0 \rightarrow 5 \rightarrow \mathbf{8} \rightarrow 6 \rightarrow 0\end{array}$ & $2,899.88$ & 0.5006 \\
\hline 12 & $\begin{array}{c}0 \rightarrow 3 \rightarrow \mathbf{1} \rightarrow 0 \\
0 \rightarrow 3 \rightarrow \mathbf{2} \rightarrow 3 \rightarrow 0 \\
0 \rightarrow \mathbf{4} \rightarrow 0 \\
0 \rightarrow 4 \rightarrow 8 \rightarrow 7 \rightarrow 5 \rightarrow 0 \\
0 \rightarrow 5 \rightarrow \mathbf{8} \rightarrow 6 \rightarrow 0\end{array}$ & $3,137.57$ & 0.4622 \\
\hline 13 & $\begin{array}{c}0 \rightarrow 3 \rightarrow \mathbf{1} \rightarrow 0 \\
0 \rightarrow 3 \rightarrow \mathbf{2} \rightarrow 3 \rightarrow 0 \\
0 \rightarrow \mathbf{4} \rightarrow 0 \\
0 \rightarrow 5 \rightarrow 8 \rightarrow 7 \rightarrow 5 \rightarrow 0 \\
0 \rightarrow 4 \rightarrow \mathbf{8} \rightarrow 6 \rightarrow 0\end{array}$ & $3,196.99$ & 0.4526 \\
\hline 14 & $\begin{array}{c}0 \rightarrow 3 \rightarrow \mathbf{1} \rightarrow 0 \\
0 \rightarrow 3 \rightarrow \mathbf{2} \rightarrow 3 \rightarrow 0 \\
0 \rightarrow \mathbf{4} \rightarrow 0 \\
0 \rightarrow 4 \rightarrow 8 \rightarrow \mathbf{7} \rightarrow 5 \rightarrow 0 \\
0 \rightarrow 4 \rightarrow \mathbf{8} \rightarrow 6 \rightarrow 0\end{array}$ & $3,434.68$ & 0.4142 \\
\hline
\end{tabular}

TABLE 5: Vehicle scheduling plan (for solution 1).

\begin{tabular}{|c|c|c|c|c|c|c|}
\hline \multirow{2}{*}{ Vehicle } & \multirow{2}{*}{ Node } & \multicolumn{5}{|c|}{ Vehicle scheduling scheme } \\
\hline & & LS & $\mathrm{DD}$ & US & RU & $\mathrm{AC}$ \\
\hline \multirow{4}{*}{1} & 7 & $6: 26$ & $7: 11$ & 8:00 & $8: 45$ & $9: 34$ \\
\hline & 1 & $9: 34$ & $10: 19$ & 11:08 & $11: 53$ & $12: 42$ \\
\hline & 8 & $12: 42$ & $13: 27$ & $14: 11$ & $14: 56$ & $15: 41$ \\
\hline & 2 & $15: 41$ & $16: 26$ & $17: 12$ & $17: 57$ & 18:42 \\
\hline \multirow{4}{*}{2} & 1 & $6: 26$ & $7: 11$ & $8: 00$ & $8: 45$ & $9: 34$ \\
\hline & 4 & $9: 34$ & $10: 19$ & $10: 59$ & $11: 44$ & $12: 25$ \\
\hline & 7 & $12: 25$ & 13:10 & $13: 59$ & $14: 44$ & $15: 33$ \\
\hline & 7 & $15: 33$ & $16: 18$ & $17: 08$ & $17: 53$ & 18:42 \\
\hline \multirow{4}{*}{3} & 7 & $6: 26$ & $7: 11$ & 8:00 & $8: 45$ & $9: 34$ \\
\hline & 1 & $9: 34$ & 10:19 & 11:08 & $11: 53$ & $12: 42$ \\
\hline & 8 & $12: 42$ & $13: 27$ & $14: 11$ & $14: 56$ & $15: 41$ \\
\hline & 2 & $15: 41$ & $16: 26$ & $17: 12$ & $17: 57$ & $18: 42$ \\
\hline \multirow{5}{*}{4} & 4 & $6: 35$ & $7: 20$ & $8: 00$ & $8: 45$ & $9: 25$ \\
\hline & 4 & $9: 25$ & $10: 10$ & $10: 51$ & $11: 36$ & $12: 16$ \\
\hline & 8 & $12: 16$ & 13:01 & $13: 46$ & $14: 31$ & $15: 15$ \\
\hline & 8 & $15: 15$ & $16: 00$ & $16: 45$ & $17: 30$ & $18: 14$ \\
\hline & 8 & 18:14 & 18:59 & $19: 44$ & $20: 29$ & $21: 13$ \\
\hline
\end{tabular}




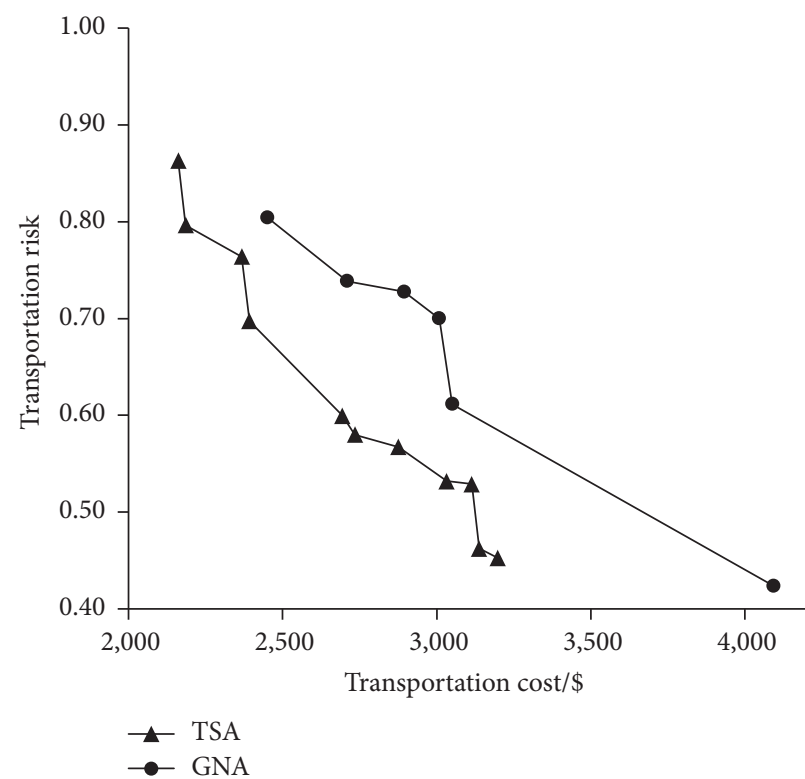

(a) Iteration number $=5$

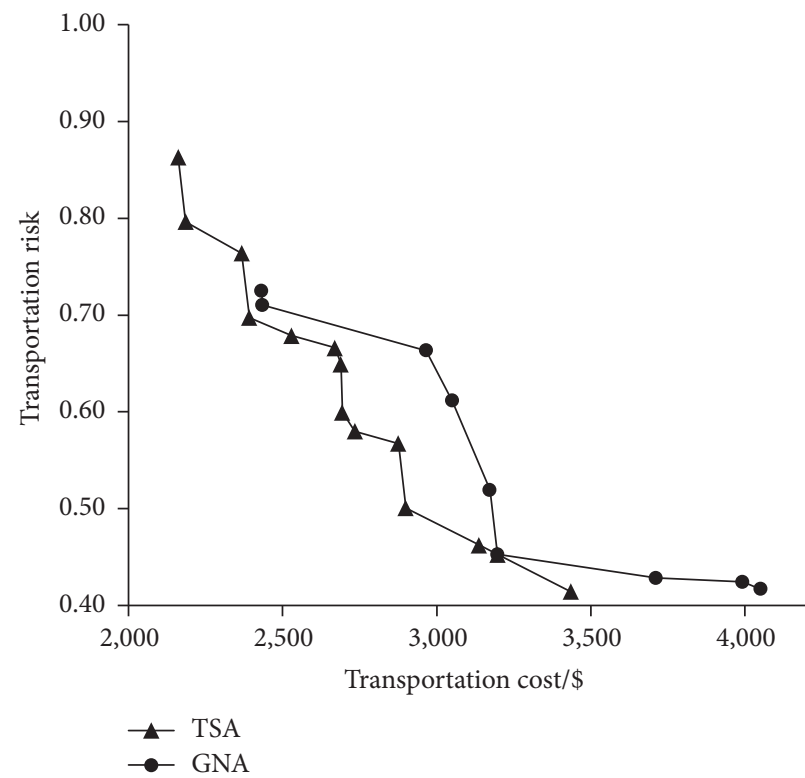

(c) Iteration number $=20$

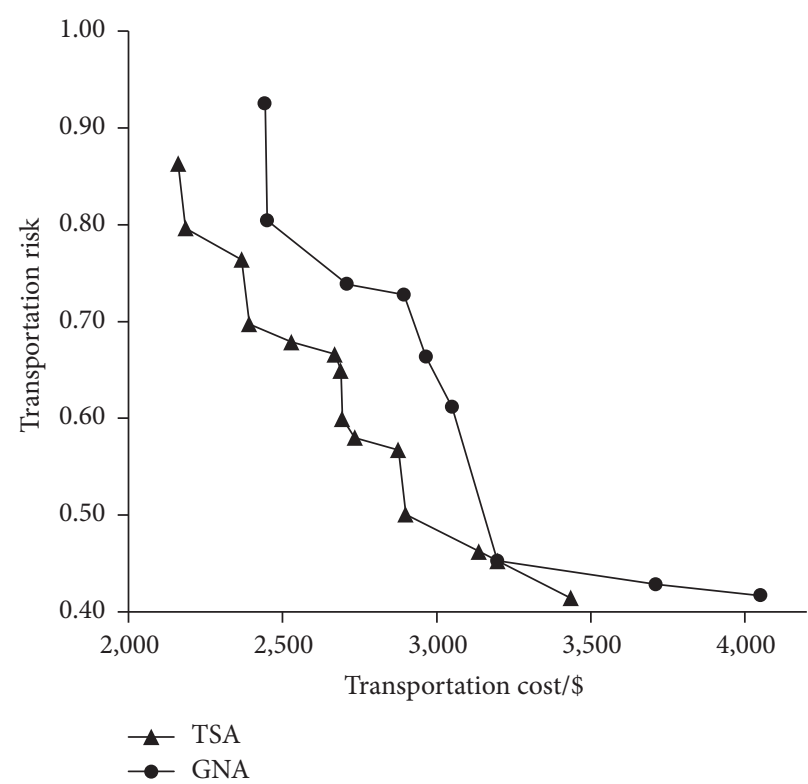

(b) Iteration number $=10$

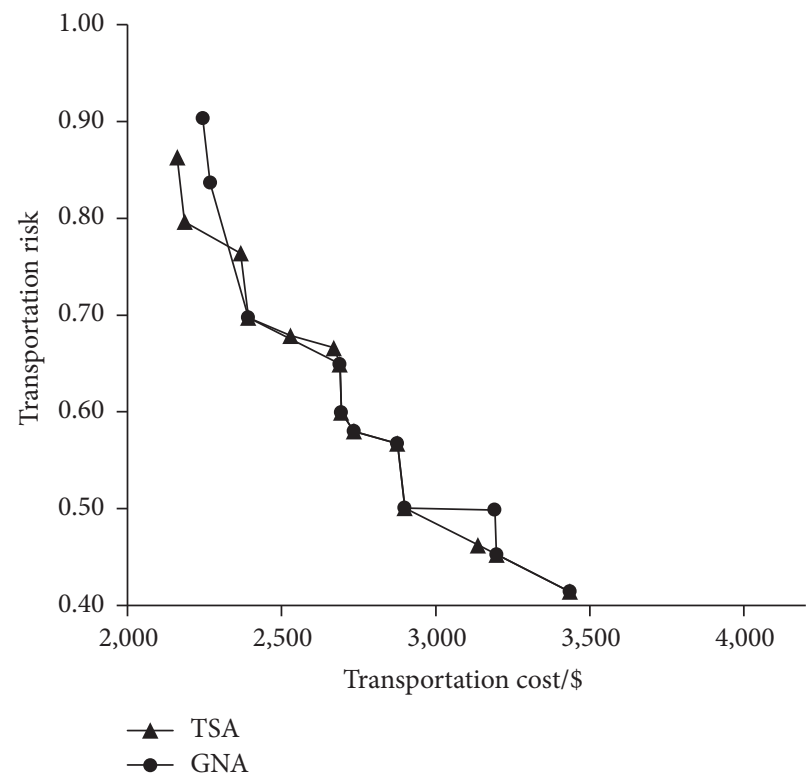

(d) Iteration number $=50$

FIgURE 6: Pareto solutions obtained in path optimization.

\section{Case Study}

The proposed methodology was implemented for the urban transportation network in Lanzhou, China. Daily traffic volumes of all road sections in realistic road networks of main districts are used to estimate vehicle travel time for all road sections. In addition, other factors such as distribution of population and facilities, as well as probabilities and consequences of traffic accident, along the road sections involved, are also considered to establish a traditional model [15] to estimate the risk value of each road section for vehicle transportation. Thus a transport network for hazardous materials (see Figure 7) in main districts of Lanzhou is formed, containing 170 nodes and 272 edges. Distribution of gasoline 92\# ( $\star$ ) for 16 gas stations $(\bullet)$ under PetroChina in this network is taken as an example to calculate vehicle scheduling plans for oil product transportation.

Table 7 shows the positions of 16 gas stations in the network, indicated by their nearest nodes (destination site $d$ ), as well as their needs of gasoline, and the time window of distribution. The position of the distribution center is indicated by node 1 . The volume of a transportation vehicle (tank car) is $13.5 \mathrm{~m}^{3}$. Traveling fee of a tank car in FCL in urban highways is $200 \mathrm{RMB} / \mathrm{h}$, while that with no load is 


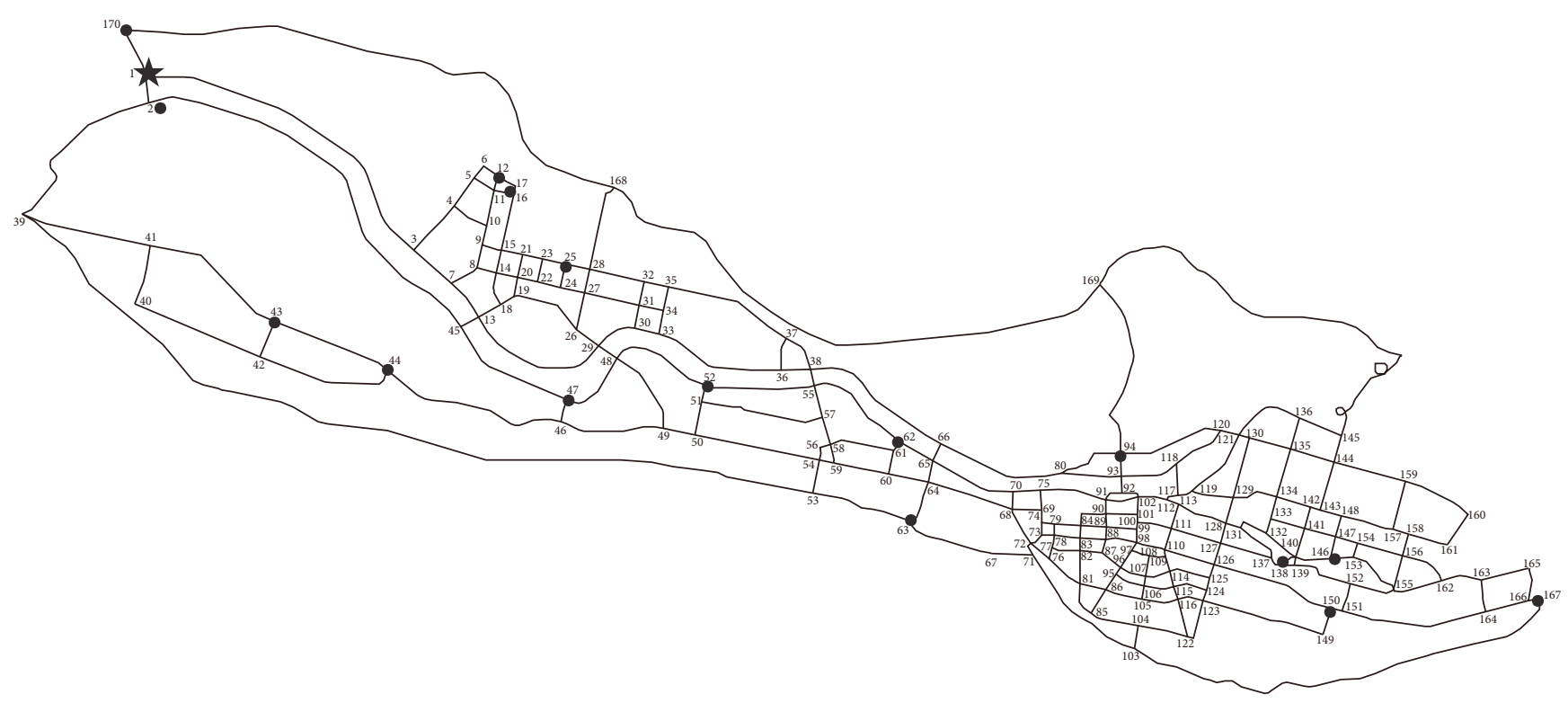

FIGURE 7: A transport network for hazardous materials in main districts of Lanzhou.

TABLE 6: Vehicle scheduling plan (for solution 14).

\begin{tabular}{|c|c|c|c|c|c|c|}
\hline \multirow{2}{*}{ Vehicle } & \multirow{2}{*}{ Node } & \multicolumn{5}{|c|}{ Vehicle scheduling scheme } \\
\hline & & LS & $\mathrm{DD}$ & US & RU & $\mathrm{AC}$ \\
\hline \multirow{3}{*}{1} & 1 & $07: 15$ & 08:00 & 08:54 & 09:39 & $10: 27$ \\
\hline & 4 & $10: 27$ & 11:12 & $11: 53$ & $12: 38$ & $13: 18$ \\
\hline & 7 & $13: 18$ & $14: 03$ & $16: 46$ & $17: 31$ & $18: 20$ \\
\hline \multirow{3}{*}{3} & 4 & $07: 15$ & 08:00 & 08:40 & 09:25 & $10: 06$ \\
\hline & 1 & $10: 06$ & $10: 51$ & $11: 44$ & $12: 29$ & $13: 18$ \\
\hline & 7 & 13:18 & 14:03 & $16: 46$ & $17: 31$ & $18: 20$ \\
\hline \multirow{3}{*}{5} & 2 & $07: 30$ & 08:15 & 09:00 & 09:45 & $10: 30$ \\
\hline & 8 & $10: 30$ & 11:15 & 13:01 & $13: 46$ & $14: 31$ \\
\hline & 8 & $14: 31$ & $15: 16$ & 17:01 & $17: 46$ & $18: 31$ \\
\hline \multirow{3}{*}{3} & 2 & $07: 30$ & $08: 15$ & 09:00 & $09: 45$ & $10: 30$ \\
\hline & 8 & $10: 30$ & 11:15 & 13:01 & $13: 46$ & $14: 31$ \\
\hline & 8 & $14: 31$ & 15:16 & 17:01 & $17: 46$ & $18: 31$ \\
\hline \multirow{3}{*}{4} & 1 & $07: 15$ & 08:00 & $08: 54$ & 09:39 & $10: 27$ \\
\hline & 4 & $10: 27$ & 11:12 & $11: 53$ & $12: 38$ & $13: 18$ \\
\hline & 7 & $13: 18$ & $14: 03$ & $16: 46$ & $17: 31$ & $18: 20$ \\
\hline \multirow{2}{*}{6} & 8 & $07: 29$ & 08:14 & $10: 00$ & $10: 45$ & $11: 30$ \\
\hline & 7 & $11: 30$ & 12:15 & $14: 57$ & $15: 42$ & $16: 31$ \\
\hline
\end{tabular}

$150 \mathrm{RMB} / \mathrm{h}$, eliminating cost of loading and unloading. Time for either loading or unloading is $0.75 \mathrm{~h}$.

$\mathrm{C \#}$ is the programming language to implement the algorithm. The running environment is $\mathrm{PC}$ i5-3470 $3.20 \mathrm{GHz}, 4 \mathrm{G}$. Parameters setting in the program and CPU times of vehicles are shown in Table 8 . A total of 164 path selection plans are obtained. When a certain plan is selected, the corresponding timetable for vehicle operation can be obtained by the vehicle scheduling program.

A path plan is a choice based on game between transportation cost and risk by a decision-maker with a specific risk preference. For example, in plan 1, which has the lowest transportation cost but the highest transportation risk, the transportation time is $4,396.58 \mathrm{RMB}$, while the transportation risk is $3.2262 ; 8$ vehicles are needed to complete jobs. As another example, in plan 164, which has the lowest transportation risk but the highest transportation cost, the transportation time is $4,609.83 \mathrm{RMB}$, while the transportation risk is 2.5586; also, 8 vehicles are needed to complete jobs. The traveling path and transport timetable for all vehicles in plan 164 are shown in Tables 9 and 10. 
TABLE 7: Nodes representing 16 gas stations, as well as their needs of gasoline, and the time window of distribution.

\begin{tabular}{lccc}
\hline Number & Destination & Required volume $/ \mathrm{m}^{3}$ & Time window \\
\hline 1 & 2 & 15 & {$[12: 00,16: 00]$} \\
2 & 12 & 26 & {$[8: 30,13: 30]$} \\
3 & 16 & 12 & {$[10: 30,16: 00]$} \\
4 & 25 & 16 & {$[8: 00,12: 00]$} \\
5 & 43 & 16 & {$[8: 00,16: 00]$} \\
6 & 44 & 29 & {$[10: 00,16: 00]$} \\
7 & 47 & 32 & {$[9: 00,15: 00]$} \\
8 & 52 & 25 & {$[9: 00,12: 00]$} \\
9 & 62 & 24 & {$[8: 00,10: 00]$} \\
10 & 63 & 10 & {$[8: 00,15: 00]$} \\
11 & 94 & 18 & {$[14: 00,18: 00]$} \\
12 & 138 & 25 & {$[8: 00,15: 00]$} \\
13 & 146 & 40 & {$[12: 00,18: 00]$} \\
14 & 150 & 23 & {$[10: 00,16: 00]$} \\
15 & 167 & 9 & {$[8: 00,16: 00]$} \\
16 & 170 & 25 & \\
\hline
\end{tabular}

TABLE 8: Transport parameters setting in the program and CPU times.

\begin{tabular}{lcc}
\hline Stage & $\begin{array}{c}\text { Parameters setting } \\
\text { Iteration }\end{array}$ & CPU times/s \\
\hline Path selection & - & - \\
Stage 1: the pulse algorithm is used to obtain the Pareto solutions of all paths & 500 & 17 \\
from the distribution center to each destination & & 32 \\
Stage 2: get all paths selection solution by NSGA-II algorithm & 200 & 100 \\
\hline Vehicle scheduling & & 9 \\
The vehicle scheduling plan under the current path selection plan is acquired by \\
UMDA
\end{tabular}

\section{Conclusion}

Distribution of hazardous materials in FCL is essentially a multistage combinatorial optimization problem. In this study, a decision approach for distribution of hazardous materials in FCL with a single distribution center is formed, which can provide a decision support for transportation enterprises and administrative authorities.

In this study, mathematical models for path planning and vehicle scheduling problems are established, respectively, with corresponding problem-solving algorithms designed. In order to solve the path planning model, a two-stage algorithm is designed. Based on screening of paths in the first stage, the computational complexity of the second stage involved in the global path optimization is significantly reduced, which improves calculation efficiency. In obtaining the vehicle scheduling plan, the operating process of a transporting vehicle is converted to single objective VRPTW. Then the problem-solving method for VRPTW on the basis of estimation of distribution is adopted to obtain the timetable for all vehicles.

The proposed approach was applied to a hypothetical numerical example, as well as an application to a real case in distribution of gasoline of Lanzhou, China. The numerical example showed that, for small-scale problems, the two-stage algorithm can obtain Pareto-optimal front faster in the same iteration. However, for large-scale problems, due to the length of coding, it is difficult to obtain Pareto-optimal path set in a limited iterative step; in the second stage, the problem size of VRPTW is only related to the number of destination nodes, not to the scale of the transportation network. Therefore, the vehicle scheduling method has high efficiency, whether it is small-scale or large-scale transportation network.

Our purpose is to design a decision support system for path planning and vehicle scheduling of distribution of hazardous materials in FCL. Further research has to be done at least in two directions: distribution of hazardous materials in FCL with multiple distribution centers and transportation with different volumes vehicle will be focused on.

\section{Conflicts of Interest}

The authors declare no conflicts of interest.

\section{Acknowledgments}

This research was jointly supported by the National Natural Science Foundation of China (nos. 61064012, 61364026, 


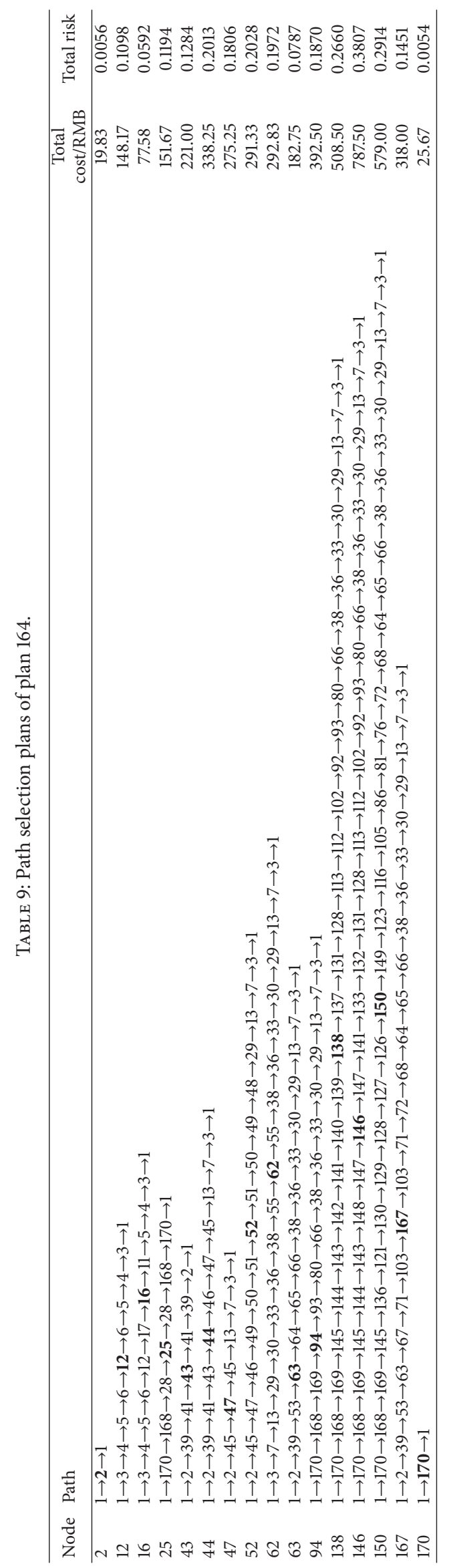


TABLE 10: Vehicle scheduling plan of plan 164.

\begin{tabular}{|c|c|c|c|c|c|c|}
\hline \multirow{2}{*}{ Vehicle } & \multirow{2}{*}{ Node } & \multicolumn{5}{|c|}{ Vehicle scheduling scheme } \\
\hline & & LS & $\mathrm{DD}$ & US & RU & $\mathrm{AC}$ \\
\hline \multirow{4}{*}{1} & 47 & 07:58 & $08: 43$ & 09:00 & 09:45 & 10:00 \\
\hline & 12 & $10: 00$ & $10: 45$ & $10: 58$ & $11: 43$ & $11: 55$ \\
\hline & 138 & $11: 55$ & $12: 40$ & $13: 29$ & $14: 14$ & $14: 54$ \\
\hline & 94 & $14: 54$ & $15: 39$ & $16: 14$ & $16: 59$ & $17: 32$ \\
\hline \multirow{4}{*}{2} & 47 & 07:58 & $08: 43$ & 09:00 & 09:45 & $10: 00$ \\
\hline & 150 & $10: 00$ & $10: 45$ & $11: 42$ & $12: 27$ & $13: 11$ \\
\hline & 2 & 13:11 & 13:56 & $13: 58$ & $14: 43$ & $14: 45$ \\
\hline & 94 & $14: 45$ & $15: 30$ & $16: 05$ & $16: 50$ & $17: 22$ \\
\hline \multirow{4}{*}{3} & 25 & $07: 15$ & 08:00 & $08: 21$ & 09:06 & 09:21 \\
\hline & 47 & 09:21 & $10: 06$ & $10: 23$ & 11:08 & $11: 23$ \\
\hline & 167 & $11: 23$ & $12: 08$ & $13: 07$ & $13: 52$ & $14: 43$ \\
\hline & 44 & $14: 43$ & $15: 28$ & $15: 49$ & $16: 34$ & $16: 55$ \\
\hline \multirow{4}{*}{4} & 43 & 07:15 & 08:00 & 08:17 & 09:02 & 09:19 \\
\hline & 52 & 09:19 & 10:04 & $10: 31$ & 11:16 & $11: 40$ \\
\hline & 138 & $11: 40$ & $12: 25$ & $13: 13$ & $13: 58$ & $14: 38$ \\
\hline & 44 & $14: 38$ & $15: 23$ & $15: 44$ & $16: 30$ & $16: 51$ \\
\hline \multirow{4}{*}{5} & 62 & $07: 15$ & 08:00 & $08: 25$ & 09:10 & 09:35 \\
\hline & 63 & 09:35 & $10: 20$ & $10: 56$ & $11: 41$ & $12: 09$ \\
\hline & 2 & $12: 09$ & $12: 54$ & $12: 56$ & $13: 41$ & $13: 42$ \\
\hline & 146 & $13: 42$ & $14: 27$ & $15: 14$ & $15: 59$ & $16: 43$ \\
\hline \multirow{4}{*}{6} & 62 & $07: 15$ & 08:00 & $08: 25$ & 09:10 & 09:35 \\
\hline & 52 & 09:35 & $10: 20$ & $10: 47$ & $11: 32$ & $11: 56$ \\
\hline & 44 & $11: 56$ & $12: 41$ & $13: 02$ & $13: 47$ & $14: 08$ \\
\hline & 146 & $14: 08$ & $14: 53$ & $15: 40$ & $16: 25$ & 17:09 \\
\hline \multirow{5}{*}{7} & 170 & $07: 15$ & 08:00 & 08:02 & $08: 47$ & $08: 49$ \\
\hline & 25 & 08:49 & 09:34 & 09:56 & $10: 41$ & $10: 56$ \\
\hline & 12 & $10: 56$ & 11:41 & $11: 53$ & $12: 38$ & $12: 51$ \\
\hline & 43 & $12: 51$ & $13: 36$ & $13: 53$ & $14: 38$ & $14: 55$ \\
\hline & 16 & $14: 55$ & $15: 40$ & $15: 54$ & $16: 39$ & $16: 52$ \\
\hline \multirow{3}{*}{8} & 170 & 07:15 & 08:00 & 08:02 & $08: 47$ & 08:49 \\
\hline & 146 & 08:49 & 09:34 & $12: 00$ & $12: 45$ & $13: 29$ \\
\hline & 150 & $13: 29$ & $14: 14$ & $15: 10$ & $15: 55$ & $16: 40$ \\
\hline
\end{tabular}

and 51408288), Natural Science Foundation of Gansu (1610RJZA048, 1610RJZA037), Youth Foundation of Lanzhou Jiaotong University (2015026), and LZJTU (201604) EP.

\section{References}

[1] M. O. Ball, B. L. Golden, A. A. Assad, and L. D. Bodin, "Planning for Truck Fleet Size in the Presence of a Common-Carrier Option," Decision Sciences, vol. 14, no. 1, pp. 103-120, 1983.

[2] A. Imai, E. Nishimura, and J. Current, "A Lagrangian relaxationbased heuristic for the vehicle routing with full container load," European Journal of Operational Research, vol. 176, no. 1, pp. 87105, 2007.

[3] J. J. Bartholdi III and K. R. Gue, "Reducing labor costs in an LTL crossdocking terminal," Operations Research, vol. 48, no. 6, pp. 823-832, 2000.

[4] N. Jozefowiez, F. Semet, and E.-G. Talbi, "Multi-objective vehicle routing problems," European Journal of Operational Research, vol. 189, no. 2, pp. 293-309, 2008.
[5] K. G. Zografos and K. N. Androutsopoulos, "A decision support system for integrated hazardous materials routing and emergency response decisions," Transportation Research Part C: Emerging Technologies, vol. 16, no. 6, pp. 684-703, 2008.

[6] R. Pradhananga, S. Hanaoka, and W. Sattayaprasert, "Optimisation model for hazardous material transport routing in Thailand," in Proceedings of the International Journal of Logistics Systems \& Management, vol. 9, pp. 22-42, 2011.

[7] R. Pradhananga, E. Taniguchi, T. Yamada, and A. G. Qureshi, "Bi-objective decision support system for routing and scheduling of hazardous materials," Socio-Economic Planning Sciences, vol. 48, no. 2, pp. 135-148, 2014.

[8] A. Raith and M. Ehrgott, "A comparison of solution strategies for biobjective shortest path problems," Computers \& Operations Research, vol. 36, no. 4, pp. 1299-1331, 2009.

[9] S. Demeyer, J. Goedgebeur, P. Audenaert, M. Pickavet, and P. Demeester, "Speeding up Martins' algorithm for multiple objective shortest path problems," $4 O R$, vol. 11, no. 4 , pp. 323348, 2013.

[10] J. C. Clímaco and M. M. Pascoal, "Multicriteria path and tree problems: discussion on exact algorithms and applications," International Transactions in Operational Research, vol. 19, no. 1-2, pp. 63-98, 2012.

[11] D. Duque, L. Lozano, and A. L. Medaglia, "An exact method for the biobjective shortest path problem for large-scale road networks," European Journal of Operational Research, vol. 242, no. 3, pp. 788-797, 2015.

[12] K. Deb, A. Pratap, S. Agarwal, and T. Meyarivan, "A fast and elitist multiobjective genetic algorithm: NSGA-II," IEEE Transactions on Evolutionary Computation, vol. 6, no. 2, pp. 182-197, 2002.

[13] H. Chai, R. C. He, C. X. Ma, and C. J. Dai, "A univariate marginal distribution algorithm hybridized with insertion heuristics for the vehicle routing problem with hard time windows," Journal of Transportation Systems Engineering and Information Technology, vol. 16, no. 2, pp. 176-182, 2016.

[14] C. Chitra and P. Subbaraj, "A nondominated sorting genetic algorithm solution for shortest path routing problem in computer networks," Expert Systems with Applications, vol. 39, no. 1, pp. 1518-1525, 2012.

[15] R. Batta and S. S. Chiu, "Optimal obnoxious paths on a network: Transportation of hazardous materials," Operations Research, vol. 36, no. 1, pp. 84-92, 1988. 


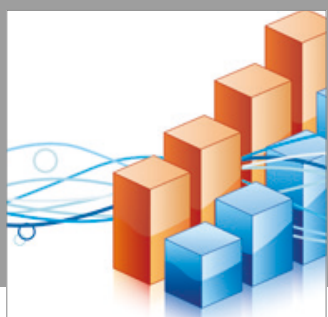

Advances in

Operations Research

vatersals

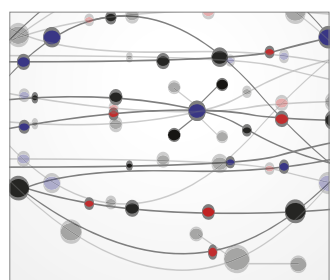

\section{The Scientific} World Journal
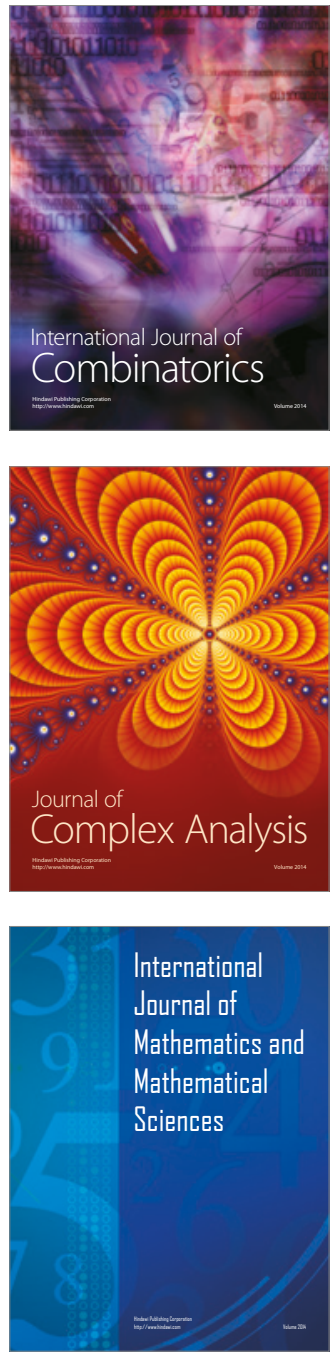
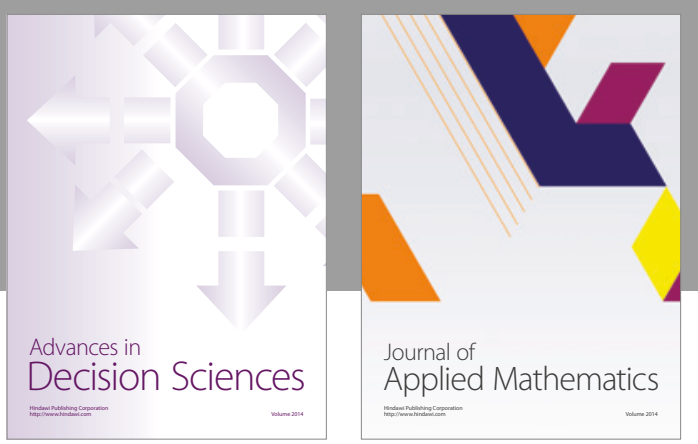

Algebra

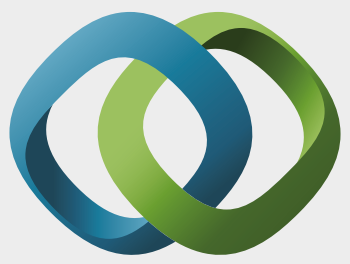

\section{Hindawi}

Submit your manuscripts at

https://www.hindawi.com
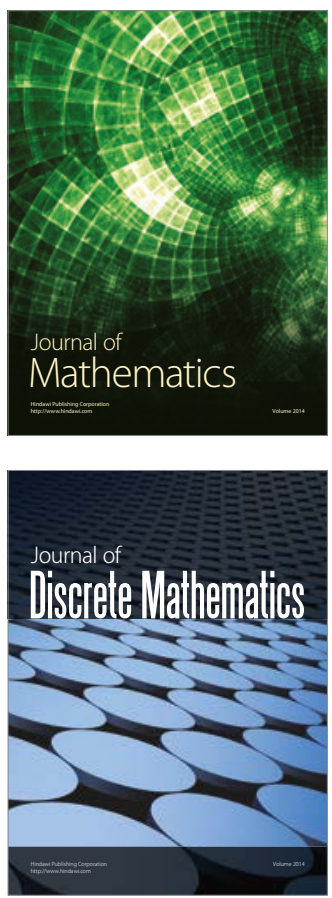

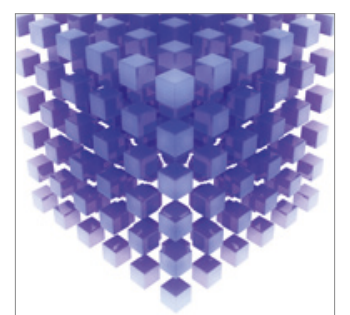

Mathematical Problems in Engineering
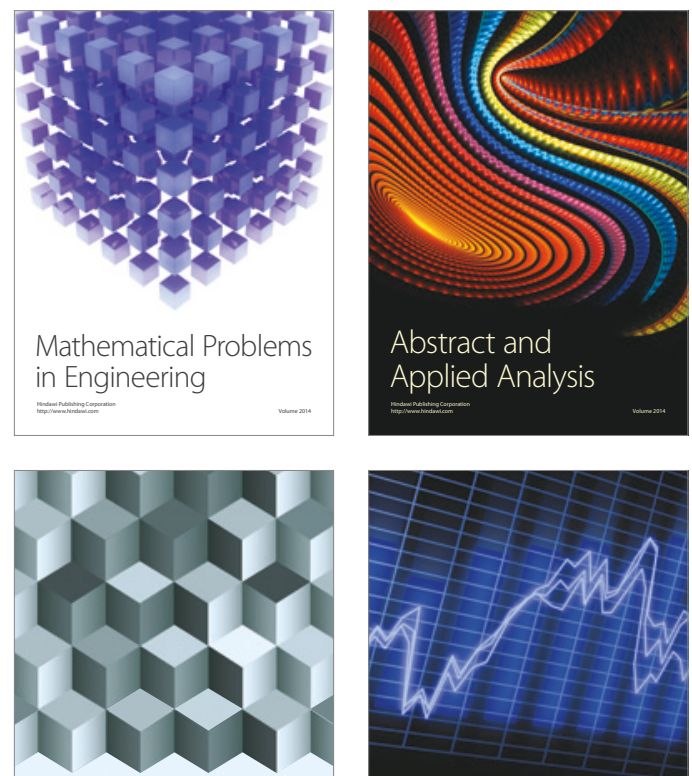

Journal of

Function Spaces

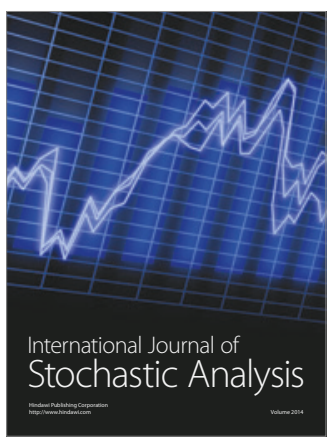

Probability and Statistics
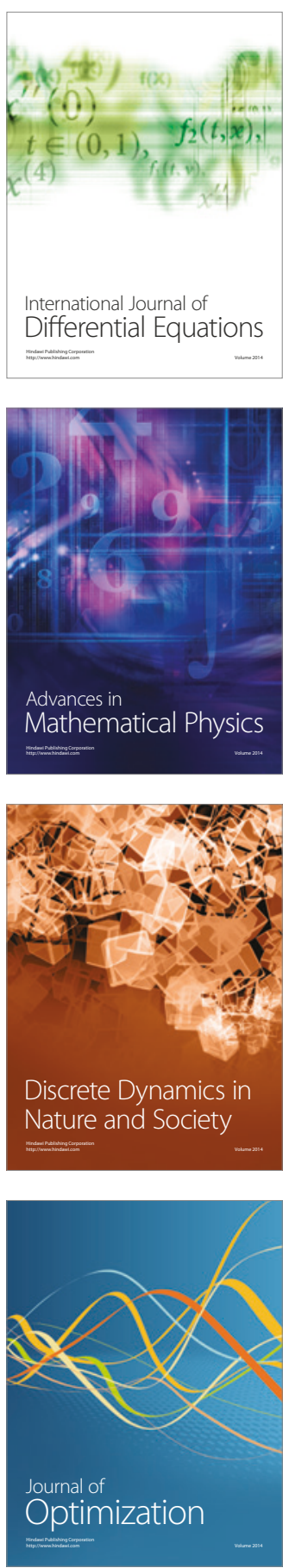